\title{
Controlling shear band instability by nanoscale heterogeneities in metallic nanoglasses
}

\author{
Sree Harsha Nandam ${ }^{1, a)}\left(\mathbb{0}\right.$, Ruth Schwaiger ${ }^{2,3,4, a)}$, Aaron Kobler $^{1}$, Christian Kübel ${ }^{1,4,5}$, \\ Chaomin Wang ${ }^{6}$, Yulia Ivanisenko ${ }^{1}$, Horst Hahn ${ }^{1,7}$ \\ ${ }^{1}$ Institute of Nanotechnology, Karlsruhe Institute of Technology, Hermann-von-Helmholtz-Platz 1, 76344 Eggenstein-Leopoldshafen, Germany \\ ${ }^{2}$ Institute for Applied Materials (IAM), Karlsruhe Institute of Technology (KIT), Hermann-von-Helmholtz-Platz 1, 76344 Eggenstein-Leopoldshafen, \\ Germany \\ ${ }^{3}$ Institute of Energy and Climate Research (IEK-2), Forschungszentrum Juelich GmbH, 52425 Juelich, Germany \\ ${ }^{4}$ Karlsruhe Nano Micro Facility, Karlsruhe Institute of Technology, Hermann-von-Helmholtz-Platz 1, 76344 Eggenstein-Leopoldshafen, Germany \\ ${ }^{5}$ Fachbereich Material- und Geowissenschaften, Fachgebiet Materialmodellierung, Technische Universität Darmstadt, Otto-Berndt-Str. 3, \\ 64287 Darmstadt, Germany \\ ${ }^{6}$ Institute of Applied Physics, Jiangxi Academy of Sciences, Nanchang 330012, China \\ ${ }^{7}$ Herbert Gleiter Institute of Nanosciences, Nanjing University of Science and Technology, Nanjing 210094, China \\ a) Address all correspondence to these authors. e-mails: sree.nandam@kit.edu; r.schwaiger@fz-juelich.de \\ Received: 15 March 2021; accepted: 22 June 2021; published online: 8 July 2021
}

\begin{abstract}
Strain localization during plastic deformation drastically reduces the shear band stability in metallic glasses, ultimately leading to catastrophic failure. Therefore, improving the plasticity of metallic glasses has been a long-standing goal for several decades. In this regard, nanoglass, a novel type of metallic glass, has been proposed to exhibit differences in short and medium range order at the interfacial regions, which could promote the formation of shear transformation zones. In the present work, by introducing heterogeneities at the nanoscale, both crystalline and amorphous, significant improvements in plasticity are realized in micro-compression tests. Both amorphous and crystalline dispersions resulted in smaller strain bursts during plastic deformation. The yield strength is found to increase significantly in $\mathrm{Cu}-\mathrm{Zr}$ nanoglasses compared to the corresponding conventional metallic glasses. The reasons for the mechanical behavior and the importance of nanoscale dispersions to tailor the properties is discussed in detail.
\end{abstract}

\section{Introduction}

Metallic nanoglasses are an emerging class of amorphous materials characterized by glassy core regions surrounded by interfaces, similar to grain boundaries in crystalline materials [1-4]. Such interfacial regions distributed at a length scale on the order of 5-10 $\mathrm{nm}$, are characterized by a different chemistry and enhanced free volume compared to the core of the glassy grains. Tailoring the amorphous structure in this way results in distinct physical properties compared to conventional metallic glasses synthesized by liquid quenching routes. Metallic nanoglasses have already shown some interesting and promising properties. Properties like ferromagnetism, enhanced bio-compatability, improved glucose detection and increased thermal stability are some of the properties improved in nanoglasses when compared to the metallic glasses of a similar composition [5-11]. However, the role of the interfacial structure on these properties seems to elude us until now.

Unsurprisingly, the presence of modified short-range order at the interfaces also affects mechanical properties such as plasticity and yield strength in nanoglasses. $\mathrm{Sc}_{75} \mathrm{Fe}_{25}$ nanoglasses showed more plastic deformation in uniaxial micro-pillar compression tests compared to the conventional metallic glasses for pillar diameters less than $300 \mathrm{~nm}$. [12]. Along with compression, even tensile plasticity was observed in the same nanoglass for a sample size of $400 \mathrm{~nm}$ [13] with a significant amount of necking during tensile deformation. However, no improvement in plasticity was observed in $\mathrm{Pd}-\mathrm{Si}$ nanoglasses compared to the corresponding melt-spun ribbons [11]. Similar results were also obtained in $\mathrm{Ni}-\mathrm{P}$ nanoglasses prepared by electrodeposition [14]. An interesting observation here is that the effect of 
chemical segregation is found to be negligible in both $\mathrm{Pd}-\mathrm{Si}$ and $\mathrm{Ni}-\mathrm{P}$ nanoglasses. Improved plasticity was also reported in $\mathrm{Fe}_{90} \mathrm{Sc}_{10}$ nanocomposites where Fe nanocrystals were found to be distributed in the amorphous matrix [15]. Besides plasticity, significant variation of the yield strength was also reported in literature, with $\mathrm{Cu}-\mathrm{Zr}$ and $\mathrm{Sc}_{75} \mathrm{Fe}_{25}$ nanoglasses showing higher yield strength during nanoindentation, while $\mathrm{Pd}-\mathrm{Si}$ and $\mathrm{Ni}-\mathrm{P}$ showed lower yield strength during micro-pillar tests compared to their conventional amorphous counterparts $[10,11,14,16]$.

Despite the work presented on the mechanical behavior of nanoglasses, several ambiguities still remain unanswered in literature. Till now, to the authors' knowledge, except $\mathrm{Sc}_{75} \mathrm{Fe}_{25}$ nanoglasses, plasticity improvement is not shown in other alloys and even in Sc-Fe system, the improvement in plasticity is only substantial in samples below $300 \mathrm{~nm}$. In view of this, we would like to clarify if the plasticity enhancement is possible in other alloy systems as well. We would also try to shed some light on the reasons for the improvement in mechanical behavior of nanoglasses. For this purpose, we have taken into consideration two alloy systems with crystalline and amorphous heterogeneities i.e., $\mathrm{Cu}-\mathrm{Zr}$, where amorphous heterogeneities were present [10] and $\mathrm{Fe}-\mathrm{Sc}$, where crystalline dispersions were revealed by advanced characterization tools like atom probe tomography (APT) and field ion microscopy (FIM) [15]. To this end, micro-compression tests on $\mathrm{Cu}-\mathrm{Zr}$ and $\mathrm{Fe}-\mathrm{Sc}$ nanoglasses/composites are performed and compared with conventional metallic glass of the same composition. The reasons for the experimental observations in the two nanoglasses will be addressed in the current report.

\section{Results}

\section{Cu-Zr nanoglasses}

The amorphous nature of the consolidated $\mathrm{Cu}_{50} \mathrm{Zr}_{50}$ nanoglass was verified by XRD. Transmission electron microscopy (TEM) was performed to reveal the microstructural features of the sample as shown in Fig. 1a and b. As shown in Fig. 1b, the high angle annular dark field (HAADF) image showed a uniform microstructure with very little contrast. The dark regions in the image indicate the residual pores present in the TEM sample. In order to observe the presence of chemical variation in the nanoglass, atom probe tomography (APT) was performed and fluctuations in the size range of 5-10 nm was observed in the $\mathrm{Cu}-\mathrm{Zr}$ nanoglass as shown in Fig. 1c. This result fits to the chemical inhomogeneity observed in a $\mathrm{Cu}-\mathrm{Zr}$ nanoglass in molecular dynamic (MD) simulations, where $\sim 10$ at.\% segregation was noticed between the core and the interface regions [17]. APT investigations carried out on
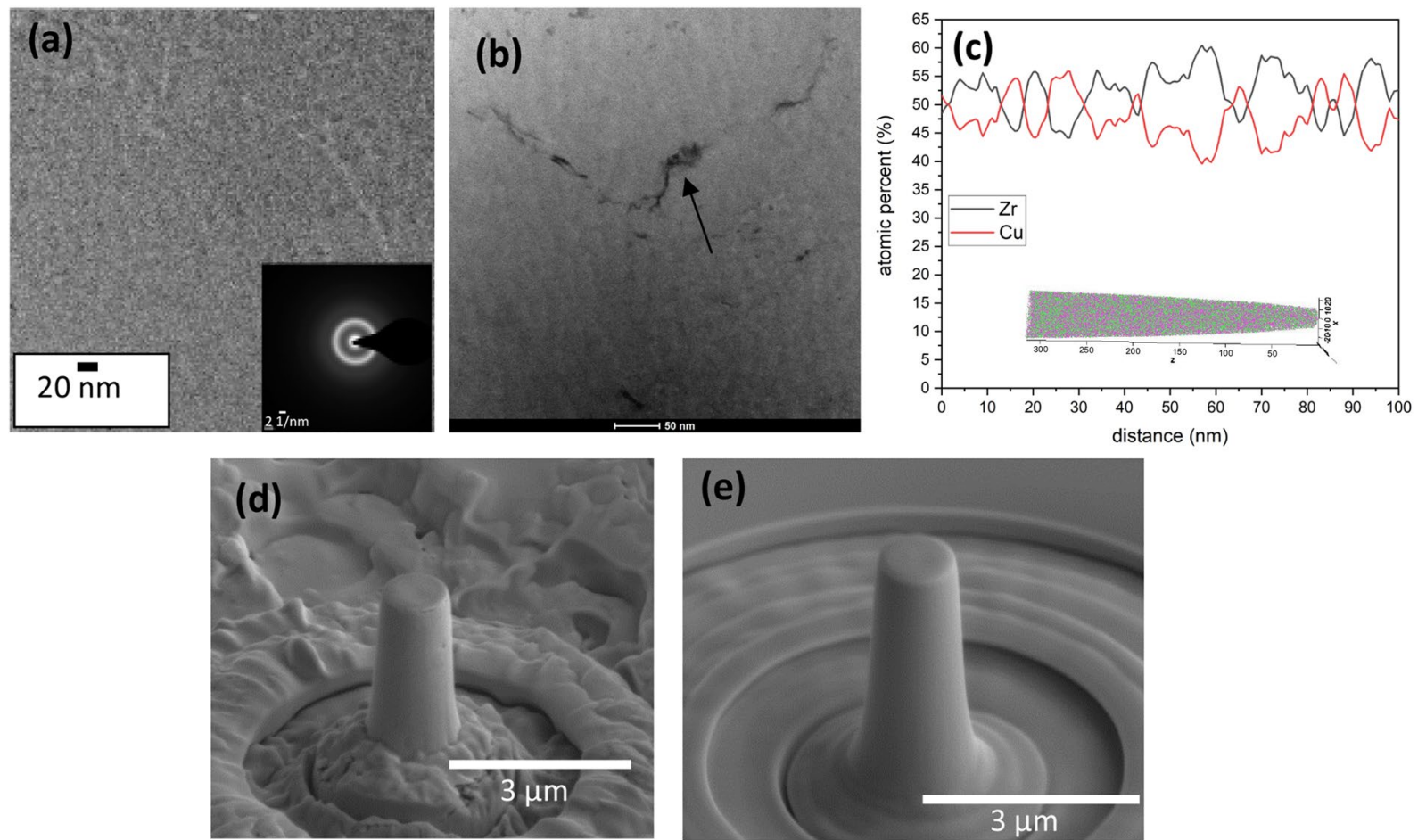

Figure 1: (a) TEM image of $\mathrm{Cu}_{50} \mathrm{Zr}_{50}$ nanoglass. Inset shows a selected area electron diffraction pattern with the diffused halo confirming the amorphous nature of the sample. (b) STEM-HAADF image showing the $\mathrm{Cu}-\mathrm{Zr}$ nanoglass, arrow indicates a porous region. (c) Composition profile along the length of the APT tip (shown in the inset) obtained using a cylinder of diameter $3 \mathrm{~mm}$. Micro-pillars of (d) nanoglass and (e) metallic glass before compression imaged by SEM. 
$\mathrm{Cu}-\mathrm{Zr}$ metallic glasses showed no such chemical segregation at the nanometer scale [18].

Uni-axial compression was performed on the micron-sized pillars shown in Fig. 1d and e to understand the deformation behavior. The displacement excursions in the stress-strain curves resulted from the serrated flow behavior and are typical of a load-controlled experiment. Engineering stress was calculated considering the diameter of the top of the pillar and engineering strain was calculated considering the portion of the pillar where the taper is less than $3^{\circ}$, neglecting the bottom portion where the diameter of the pillar increases significantly. The compliance of the material underneath the pillar is subtracted applying Sneddon's correction [19]. The elastic modulus obtained from the nanoindentation data [10] is used for correcting the compliance of the substrate. Note that at higher strains, due to localized flow of the material, correct determination of the stress is very difficult. The stress-strain curves of the nanoglass and metallic glass showed an initial linear elastic response followed by plastic deformation as shown in Fig. 2. A clear onset of yielding was observed in the metallic glasses at around $\sim 2400 \mathrm{MPa}$ followed by a distinct strain burst. On the other hand, nanoglasses showed a deviation from linearity around 2700-2800 MPa beyond which the stress kept on increasing until it reaches a stress of 3100-3300 MPa as shown in the inset of Fig. 2. To make a distinction between these effects, $2 \%$ offset yield stress is taken as the yield strength and the values of all the tested conditions are presented in Table 1 . The yield strength of the pillar with pre-existing pores showed lower yield strength than the other nanoglass pillars, but still higher strength than the corresponding metallic glass (see Table 1). The effect of porosity on the plastic deformation behavior of nanoglasses will

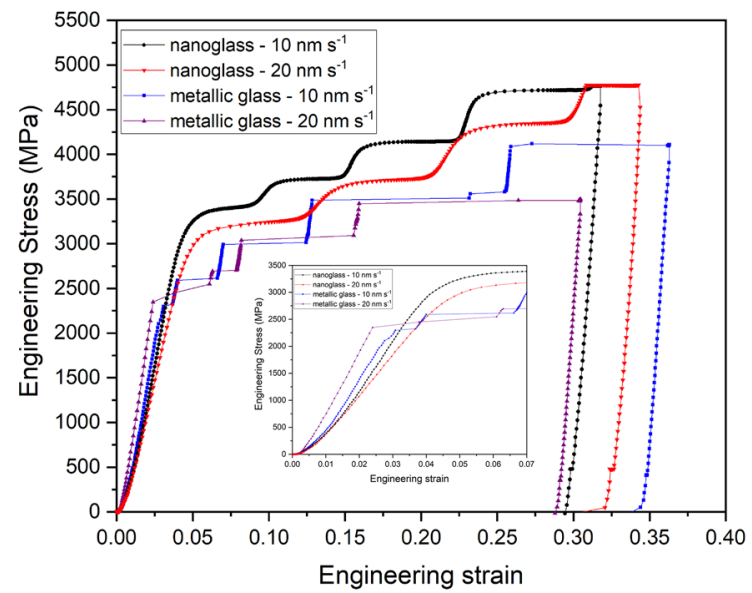

Figure 2: Engineering stress-strain curves for the nanoglass and metallic glass samples determined from micro-compression tests at a displacement rate of 10 and $20 \mathrm{~nm} \mathrm{~s}^{-1}$. Inset magnifying the elastic region of the stress-strain curve.
TABLE 1: $2 \%$ offset yield stress of $\mathrm{Cu}-\mathrm{Zr}$ nanoglass and metallic glass for all the tests.

\begin{tabular}{lcc}
\hline \hline Displacement rate in $\mathrm{nm} \mathrm{s}^{-1}$ & Nanoglass & Metallic glass \\
\hline 10 & 3317 & 2292 \\
10 & $2964^{\mathrm{a}}$ & 2089 \\
10 & 3405 & 2453 \\
20 & 3148 & 2355 \\
20 & 3781 & 2569 \\
100 & 3621 & 2409 \\
\hline \hline
\end{tabular}

All the values are in $\mathrm{MPa}$

apillar with porosity-Fig. 8a.

be discussed in 'Factors affecting the deformation behavior' section.

Besides differences in the yield strength, contrasting features are also observed in the plastic regime of the nanoglass and the metallic glass. Pronounced displacement bursts observed in the metallic glasses are absent in the nanoglasses. The data acquisition rate during the current compression test is $10 \mathrm{~Hz}$, which translates to $0.1 \mathrm{~s}$ for each data point in the stress-strain curve. In the nanoglass, several data points are recorded during each displacement burst as shown in the stress-strain curve (see Fig. 2), while no data point can be recorded during the displacement bursts observed for the metallic glass. This gives us a strong indication that the shear instability in a nanoglass is not as catastrophic as in a conventional metallic glass. All the nanoglass micro-pillars behaved in a similar way under all test conditions. Each data point in the plastic region of the nanoglass is separated by $\sim-10 \mathrm{~nm}$, while it is approximately around $100 \mathrm{~nm}$ in the metallic glass. One can make a rudimentary estimate of the strain rate during the displacement burst with the available information. Assuming all the shear displacement is localized in the shear band, strain accommodated in the shear bands is 1 , width of shear band is $\sim 10 \mathrm{~nm}$ and time taken for the shear band to traverse the distance between two data points as $0.1 \mathrm{~s}$, then shear strain rate can be estimated to be $10 \mathrm{~s}^{-1}$ in the nanoglass and $100 \mathrm{~s}^{-1}$ in the metallic glass. As a word of caution, the estimates made here provide us only with a qualitative understanding about the strain rate in a shear band, but not a realistic quantitative description. To gain a quantitative understanding of pop-in length and strain rate, one has to consider the effects of the machine control parameters on the strain bursts [20].

In a micro-compression test, especially at low strains, each displacement burst in the stress-strain curve can be directly correlated to the generation of an individual shear band [21]. Deformed pillars were then observed by SEM to gain more insight into the number of shear bands and their morphology. A clear difference can be noticed in both the number of shear 

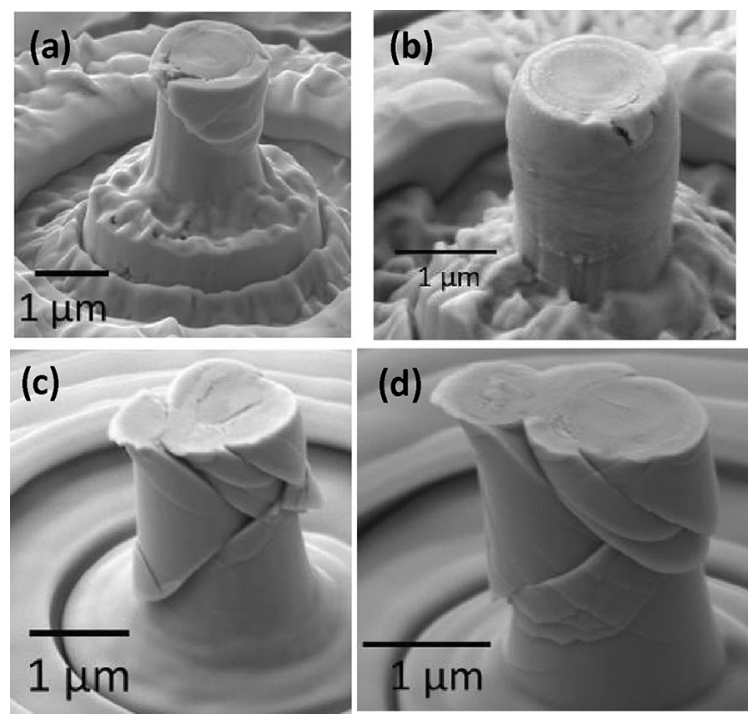

Figure 3: SEM images of deformed pillars of $\mathrm{Cu}-\mathrm{Zr}$ nanoglass deformed at (a) $10 \mathrm{~nm} \mathrm{~s}^{-1}$, (b) $20 \mathrm{~nm} \mathrm{~s}^{-1}, \mathrm{Cu}-\mathrm{Zr}$ metallic glass at a displacement rate of (c) $10 \mathrm{~nm} \mathrm{~s}^{-1}$, (d) $20 \mathrm{~nm} \mathrm{~s}^{-1}$.

bands and also their size. Compressed nanoglass pillars exhibit faint shear bands as shown in Fig. 3a and b, while the metallic glass pillars showed prominent and distinctive shear bands as illustrated in Fig. $3 \mathrm{c}$ and d. The nanoglass pillar in Fig. $3 \mathrm{~b}$ clearly does not show any major shear band and seems to deform in a homogeneous manner. To decipher if the deformation is homogeneous or not, one of the nanoglass pillars was deformed to a strain of about 0.25 and the compressed pillar showed a mushroom kind of bulge in the top portion as shown in Fig. $4 \mathrm{a}$ and $\mathrm{b}$. Earlier reports on micro-pillar studies have claimed that such mushroom kind of deformation in glassy materials is the result of homogeneous deformation [22]. However, in the present case, a closer observation of the pillar showed a number of faint shear bands as shown in Fig. $4 \mathrm{a}$ and b. At least five shear bands can be observed in the post-compressed pillars, while the corresponding stress-strain curve showed three strain bursts. To summarize, the deformation in the nanoglass proceeds in a pseudohomogeneous manner with the formation and propagation of fine shear bands.

\section{Fe-Sc nanocomposites}

To understand the deformation behavior and the influence of crystalline heterogeneity in contrast to an amorphous dispersion, we also performed micro-compression tests on $\mathrm{Fe}_{90} \mathrm{Sc}_{10}$ nanocomposites. Initial characterization of the specimen was performed by XRD and further characterization was conducted by TEM. Figure 5 a shows scanning transmission electron micrograph (STEM) images of Fe-Sc nanocomposites with bright and dark contrast regions corresponding to chemical variations and residual porosity present in the sample after the compaction

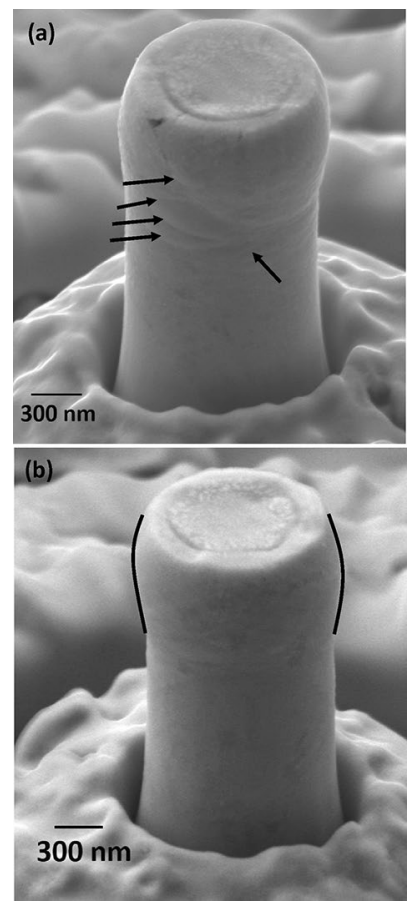

Figure 4: (a) and (b) SEM micrographs of a compressed Cu-Zr nanoglass pillar imaged from two opposite sides. Microcompression was conducted at a displacement rate of $20 \mathrm{~nm} \mathrm{~s}^{-1}$ to a maximum displacement of $500 \mathrm{~nm}$. Arrows indicate the shear bands in (a). Mushroom kind of bulge is indicated by the lines in (b).

in IGC. HRTEM images in Fig. 5b confirmed that both amorphous and crystalline phases are present in the nanoglass samples. Inset in Fig. 5b revealed an amorphous halo along with a few defined diffraction spots highlighting that a crystalline phase, i.e. $\alpha-F e$, is present in the nanoglass sample, which in the following will be referred to as nanocomposite. The exact phase fraction of the crystalline regions is estimated to be around 10 vol.\%, which was earlier confirmed by Mössbauer spectroscopy in a similar Fe-Sc nanocomposite [5]. $\alpha$-Fe is a by-product of the IGC synthesis because of the inherent difficulty in controlling the evaporation of the Fe-Sc alloy. From the characterization performed on the $\mathrm{Fe}-\mathrm{Sc}$ nanocomposites, it was concluded that they are primarily composed of interfaces, cores and $\alpha-\mathrm{Fe}$ crystallites [15]. Previous reports claimed that the segregation between core and interfaces is not very significant, only in the order of few atomic percent [23]. Crystalline regions are clearly revealed in the HRTEM images of Fig. $5 c$ and the corresponding Fast Fourier Transform (FFT) in a metallic glass composite. For the sake of clarity, we will address $\mathrm{Fe}-\mathrm{Sc}$ samples made by IGC as nanocomposites, while the $\mathrm{Fe}-\mathrm{Sc}$ melt-spun samples as metallic glass composites.

Deformation behavior of the $\mathrm{Fe}-\mathrm{Sc}$ nanocomposites showed similar features like $\mathrm{Cu}-\mathrm{Zr}$ nanoglasses as shown in Fig. 6a. The initial elastic regime is followed by an elastic limit in the nanocomposite, after which there is a deviation from the linear 

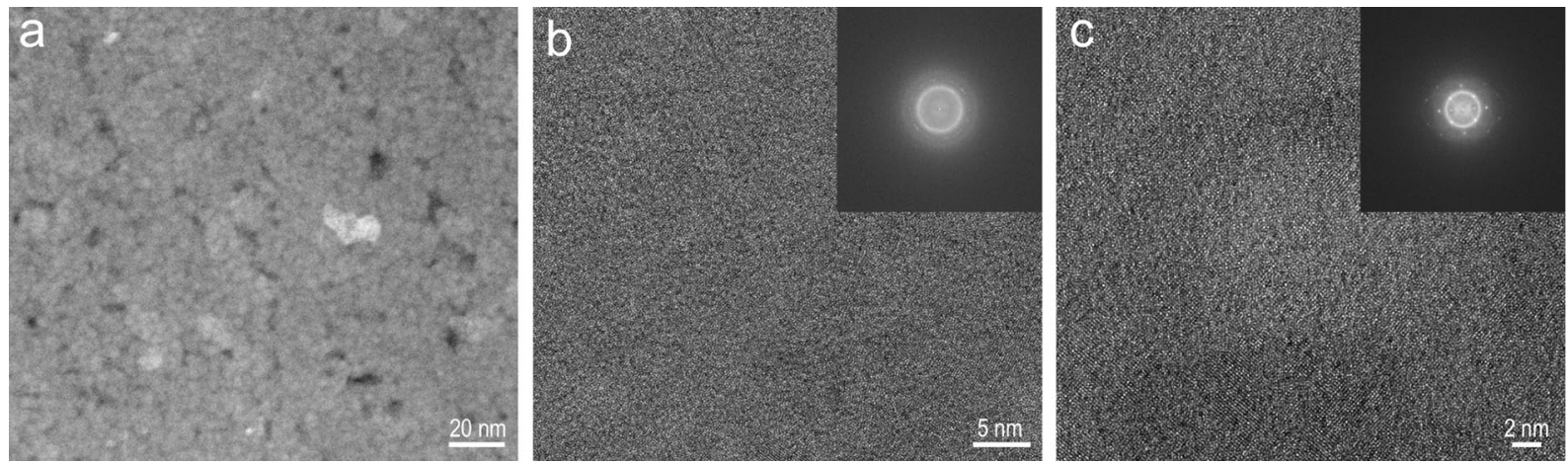

Figure 5: TEM images of $\mathrm{Fe}_{90} \mathrm{SC}_{10}$ (a) and (b) nanocomposite and (c) metallic glass composite. (a) The intensity differences in the HAADF-STEM image reveal chemical fluctuations and residual porosity in the nanoglass. The HRTEM images in (b) and (c) with the corresponding FFTs as insets exhibit the presence of crystalline phases giving rise to the defined diffraction spots in the FFTs. The FFTs correspond to the full areas of the micrographs shown.
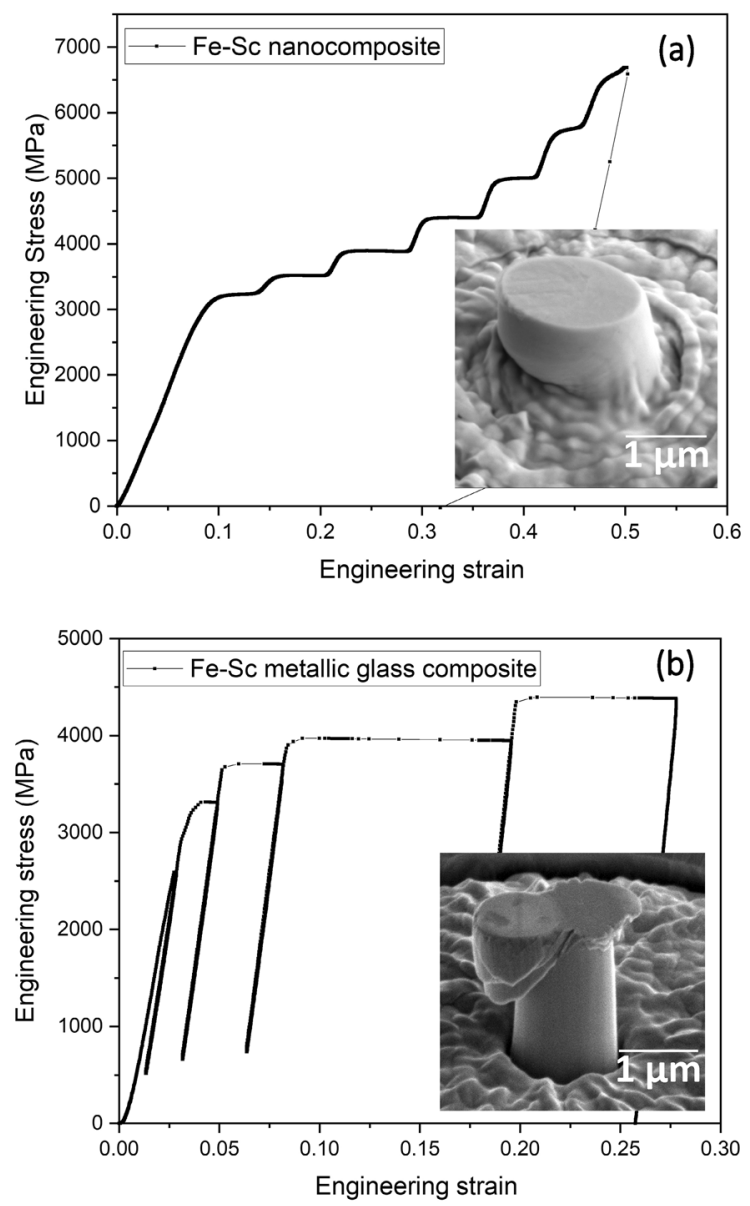

Figure 6: (a) Engineering stress-true strain curves for the micropillar compression tests of $\mathrm{Fe}_{90} \mathrm{Sc}_{10}$ nanocomposite and (b) metallic glass composite. Images in the inset show the corresponding postcompressed micro-pillars.

portion of the curve until the maximum stress is reached. Further deformation proceeded at a constant flow stress and smaller displacement bursts (see Fig. 6a). A large number of faint shear bands facilitate the deformation in the $\mathrm{Fe}-\mathrm{Sc}$ nanocomposite as shown in the inset of Fig. 6a. It is interesting to observe that the pillar deformed to very large strains, without showing any catastrophic failure. In comparison, $\mathrm{Fe}-\mathrm{Sc}$ metallic glass composites of the same composition is also tested in cyclic loading conditions and it displayed pronounced strain bursts as shown in Fig. 6b. As expected, the deformed pillars exhibited distinct shear bands representing the strain bursts as shown in the inset of Fig. $6 \mathrm{~b}$. Since it was difficult to resolve the shear bands in the nanoglass pillar compressed to high strains (inset of Fig. 6a), one of the pillars was deformed to a lower strain of 0.15 and it showed only one displacement burst as shown in the stress-strain curve of Fig. 7a. The deformed micro-pillar revealed a small mushroom kind of bulge in the top portion of the pillar similar to $\mathrm{Cu}-\mathrm{Zr}$ nanoglasses. Also, two faint and distinct shear bands were clearly observed as shown in Fig. $7 \mathrm{~b}$. Therefore, like in the case of $\mathrm{Cu}-\mathrm{Zr}$ nanoglasses, the deformation in $\mathrm{Fe}-\mathrm{Sc}$ nanocomposites is predominantly controlled by the generation and propagation of a number of shear bands.

\section{Discussion \\ Factors affecting the deformation behavior}

In the literature, several extrinsic and intrinsic factors have been discussed to be responsible for the shear band stability in bulk metallic glasses. Among the extrinsic factors, testing machine stiffness [24], strain rate [25], size of the sample [26] and temperature of the measurement [27] are found to be crucial factors influencing the deformation behavior. In the current set of experiments, all the above-mentioned extrinsic factors are maintained constant for a particular alloy system during micro-compression. Furthermore, the variation of the machine stiffness due to the two indenters used to test the Fe-Sc pillars did not show any differences. Therefore, we can conclude that the extrinsic factors do not influence the observed shear band stability in the nanoglasses/composites. The intrinsic factors responsible for 

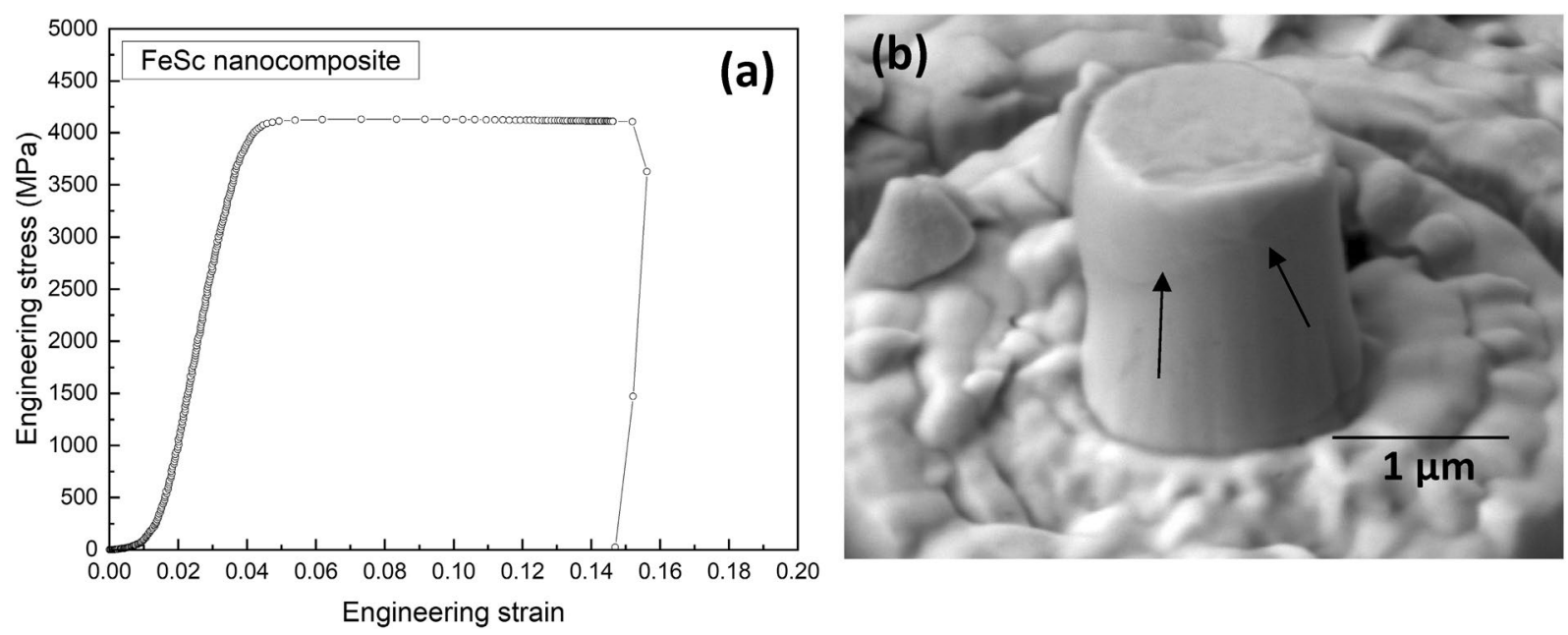

Figure 7: Engineering stress-strain curve of (a) $\mathrm{Fe}_{90} \mathrm{Sc}_{10}$ nanocomposite tested to an intermediate strain of 0.16 and (b) corresponding pillar with two major shear bands indicated by the two arrows.

the deformation behavior are residual porosity, internal micro/ nanostructure, and the presence of oxides. Presence of oxides can be ruled out because both APT measurements, done on the surface and the carrier gas hot extraction method, which is a bulk technique showed less than 2 at.\% oxygen in the nanoglass samples [10]. The samples were also stored in the glove box filled with Ar to prevent any contamination during storage. Based on the existing evidence, we can assume that the effect of oxygen may not be playing a significant role in the deformation process. The other influencing factor is the residual porosity present in the sample after the compaction in IGC. To unambiguously rule out the effect of porosity, nanoglass micro-pillars obviously containing residual porosity were tested in both $\mathrm{Cu}-\mathrm{Zr}$ and $\mathrm{Fe}-\mathrm{Sc}$ alloy systems. The micro-pillars before the tests are shown in Fig. 8a and c The stress-strain curves of the porous pillars clearly showed a brittle failure in the nanoglass/composites as shown in Fig. 8a. Both the micro-pillars after compression clearly showed distinct shear bands as shown in Fig. 8b and $\mathrm{d}$. This concludes that the observed shear band stability in nanoglasses is not due to the effect of micro or nano porosity present in the sample. Additionally, porosity should result in a reduction of the strength, while compared to the metallic glass samples, the nanoglass/composite samples showed higher offset yield stress. Therefore, we can conclude that the mechanical behavior observed in the nanoglasses/composites is a result of the inherent structure of the material.

\section{Effect of heterogeneities on the yield strength}

Yielding in a metallic glass at room temperature requires the co-operative movement and percolation of several shear transformation zones (STZ's) resulting in the formation of a shear band. Percolation of STZ's is found to be triggered by the rotation and activation of neighboring clusters in a metallic glass [28] and the activation of STZ's is largely dependent on the local atomic arrangements (or physical structure) as well as the chemical make-up of elements [29]. In $\mathrm{Cu}-\mathrm{Zr}$ nanoglasses, $\mathrm{Cu}$ and $\mathrm{Zr}$-rich zones were observed by APT in the size range of less than $10 \mathrm{~nm}$ and the compositional variation is between $\mathrm{Cu}_{45} \mathrm{Zr}_{55}$ and $\mathrm{Cu}_{57} \mathrm{Zr}_{43}$ as shown in Fig. 1c. Such compositional changes naturally result in structural changes, which may also result in the local change of elastic moduli and the yield strength. Since the exact correlation between chemistry and the free volume is not completely understood in the nanoglasses, we can discuss on the consequences of these features on the strength individually. The effect of chemistry can be understood by comparing the yield strength of $\mathrm{Cu}-\mathrm{Zr}$ metallic glasses with varying $\mathrm{Cu}$ concentrations. The bulk yield strength values of $\mathrm{Cu}_{45} \mathrm{Zr}_{55}$ and $\mathrm{Cu}_{57} \mathrm{Zr}_{43}$ metallic glasses are $\sim 1.6$ and $\sim 2 \mathrm{GPa}$, respectively [30]. The yield strength of the $\mathrm{Cu}-\mathrm{Zr}$ nanoglass is close to the value of $\mathrm{Cu}_{57} \mathrm{Zr}_{43}$ metallic glass. This result is in agreement with the data presented on bulk metallic glass (BMG) composites presented by Narayan et al. [31]. Their observations clearly highlighted that the yield strength is largely controlled by the harder phase present in the composite.

However, one should look at the above analysis with a degree of caution as we have neglected the effect of free volume and the variation of the local atomic structure in the interfacial or core regions. In fact, Hirmukhe et al. [32] have recently used finite element simulations to study the effect of free volume in the interfacial regions to understand the higher strength in $\mathrm{Sc}_{75} \mathrm{Fe}_{25}$ nanoglasses compared to metallic glasses. They have found that the pressure sensitivity of the glass-glass interfacial regions creates an additional hardening 

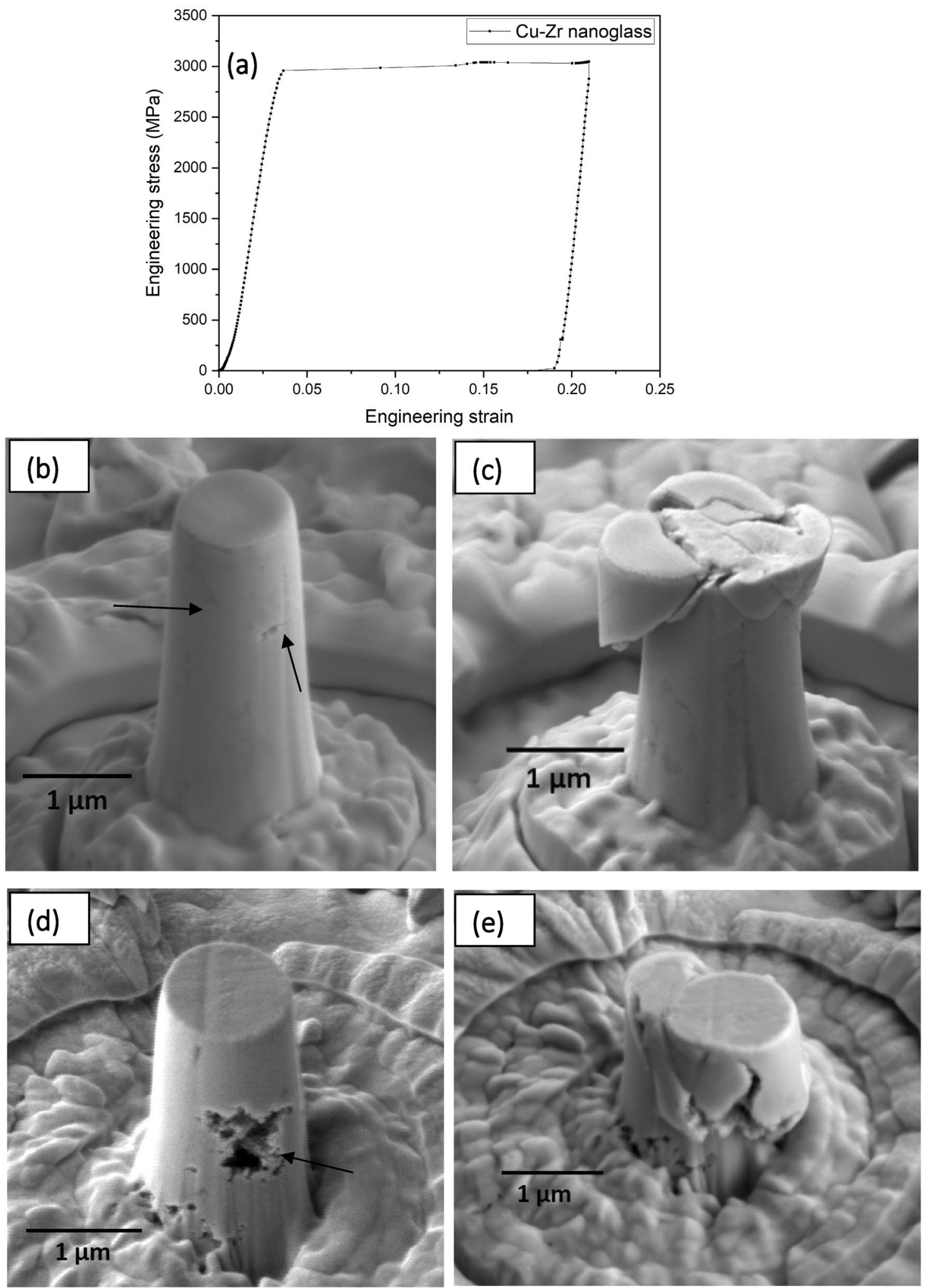

Figure 8: (a) Representative engineering stress-strain curve of $\mathrm{Cu}-\mathrm{Zr}$ nanoglass with porosity. SEM images of $\mathrm{Cu}-\mathrm{Zr}$ nanoglass pillar with porosity (b) before testing and (c) after testing, and Fe-Sc nanocomposite pillar with porosity (d) before testing and (e) after testing. Arrows in (b) and (d) denote the pores present in the pillars. 
effect. Interfacial regions, which are pockets of additional free volume densify when a stress is applied to them. In the past, a $\mathrm{Zr}$ based bulk metallic glass was found to show strain hardening because of densification due to the pressure dependent flow [33]. However, compositions like $\mathrm{Pd}-\mathrm{Si}, \mathrm{Ni}-\mathrm{P}$ nanoglasses have shown lower hardness than the bulk metallic glass compositions $[12,16]$. This leads us to conclude that the increase in strength in certain nanoglass compositions is a consequence of both chemical and structural change at the interfacial regions.

\section{Effect of heterogeneities on the plastic deformation}

Plastic deformation in metallic glasses at room temperature involves the formation of a shear band and its propagation. In general, regions of high free volume are considered to be favored nucleating sites for the formation of STZ's in amorphous materials [34]. In nanoglasses, STZs are nucleated both in the interfaces and the cores, with interfaces being the preferential nucleating sites, according to MD simulations [35]. Therefore, the stability of shear bands in nanoglasses largely depends on how the STZs percolate through the cores from the interfacial regions. It has been observed both experimentally and by MD simulations in Pd-Si nanoglasses by Nandam et al. [12], that when the chemical segregation is not significant, like in the case of Pd-Si, percolation of STZs can occur through the glassy core regions easily making the shear band formation and propagation quite straightforward. However, in nanoglasses like $\mathrm{Cu}-\mathrm{Zr}$, such a combination of STZs is difficult because of the constant change in the chemical as well as the physical structure. In other words, local changes in the chemistry inherently results in a local change in the physical structure, which induces a change in the local deformation. It consequently leads to a deflection and thus further spreading of the STZ formation and thereby a dissipation in a much wider region. Note that the critical length necessary to cause a run-away shear band failure was estimated to be $\sim 100 \mathrm{~nm}$ [36]. Since the amorphous phase is dispersed in the size range of $\sim 10 \mathrm{~nm}$ in $\mathrm{Cu}-\mathrm{Zr}$ nanoglasses, formation of a mature shear band to the size of $100 \mathrm{~nm}$ is largely delayed in nanoglasses and thus causes the deformation to proceed without catastrophic failure of the sample. Furthermore, the relative content of $\mathrm{Cu}$ or $\mathrm{Zr}$ can influence the plastic behavior of $\mathrm{Cu}-\mathrm{Zr}$ binary metallic glasses as reported both in experiments and atomistic simulations [29, 30]. Increase in $\mathrm{Zr}$ content typically results in an increase in the plasticity of $\mathrm{Cu}-\mathrm{Zr}$ binary metallic glasses.

To understand the importance of chemical segregation in terms of shear band propagation, one can look at the strain rate that is occurring in a shear band. It can be expressed according to the following equation: $\dot{\gamma}=\rho \gamma_{\mathrm{o}} v_{\mathrm{o}} \exp \left(-\left(\Delta G / K_{\mathrm{B}} T\right)\right)$, where $\rho$ is the number of sites available for STZ activation, $\gamma_{\mathrm{o}}$ is related to the strain associated in a shear band, $v_{\mathrm{o}}$ represents the characteristic frequency, $K_{\mathrm{B}}$ is the Boltzmann constant, and $T$ is room temperature (in the present case) [37]. It is evident from the equation that there is a linear correlation between the number of free volume sites and the strain rate in a shear band. Assuming the characteristic frequency is the same, then the contributing factor to decrease the shear band velocity in the nanoglass is the activation energy required for the STZ activation. It is clear from the equation that if the activation energy increases by $1 \%$, then the shear band propagation can be significantly decreased by almost $11 \%$. Since there is no available data on $\mathrm{Cu}-\mathrm{Zr}$ binary alloys regarding the STZ activation energies, we rely on the data available in ternary alloys for making an argument regarding the shear band velocity. The average activation energy for STZ formation in $\mathrm{Cu}-\mathrm{Zr}-\mathrm{Al}$ metallic glasses is found to change by almost 50\% when the $\mathrm{Zr}$ concentration is increased from 45 to 65 at.\% [38]. Thus, it is reasonable to conclude that the compositional fluctuations play a significant role in slowing down the propagation of a shear band. Such compositional inhomogeneity also delays cavitation events, which are responsible for the final fracture of the sample [39].

Similar plastic behavior is observed in $\mathrm{Fe}-\mathrm{Sc}$ nanocomposites with the crystalline dispersions reducing the chances of a run-away shear band. A probable explanation for the deformation behavior can be understood as follows. Assuming a homogeneous distribution of 10 vol.\% crystalline phase fraction [6] and the size of nanoparticle to be $10 \mathrm{~nm}$, which seems reasonable from the TEM micrographs, we can estimate the average crystalline particle spacing to be $\sim 20 \mathrm{~nm}$ in Fe-Sc nanocomposites. This interparticle spacing falls in the same range as in $\mathrm{Cu}-\mathrm{Zr}$ nanoglasses. Along with the interfacial regions between glassy cores, interfaces between the nanocrystals and the amorphous phase are also expected to promote the nucleation of STZs in the nanocomposites. It is natural to assume that the effective blocking of the shear bands by crystalline precipitates depends on their size as well as their interparticle spacing. If the precipitate size is in the same size range as the width of the shear band $(\sim 10 \mathrm{~nm})$, they can hinder the propagation of shear bands. This also comes with the constraint that the shear band should not be mature enough so that the stress field associated with the shear band simply wraps around the crystalline precipitate and moves forward [40]. In other words, the spacing between the nanocrystals should not be large so that shear bands, which may form, do not mature to a major one. In Fe-Sc nanocomposites, a particle size of $\sim 10 \mathrm{~nm}$ and a spacing of about $20 \mathrm{~nm}$ seems to be sufficient to hinder the formation of a mature shear band.

A common feature in both the $\mathrm{Cu}-\mathrm{Zr}$ nanoglasses and the $\mathrm{Fe}-\mathrm{Sc}$ nanocomposites is the number of fine shear bands carrying out the plastic deformation. This is indicative of the fact that the generation of the shear bands is the key feature in nanoglasses/composites. It is generally observed that shear bands that 
form during the plastic deformation are difficult to propagate because of either the compositional fluctuations or the crystalline dispersions and therefore in the process promote new shear bands in other regions of the sample [41, 42]. However, such a beneficial effect was not observed in the Fe-Sc metallic glass composite (present work) despite the presence of crystalline regions. This could be largely due to the higher interparticle spacing between the crystalline regions in the rapidly quenched ribbons. Another interesting feature is that the plastic regime of the present nanoglass/composite pillars resembles the one of nanocrystalline metals $[43,44]$, which showed a set of elastic loadings each followed by a softening segment. A lack of strain hardening capacity is the reasons for shear instability in nanocrystalline mechanisms. By combining amorphous clusters and crystalline dispersions, improvement in plasticity can be accomplished in nanostructured materials.

\section{Conclusions}

In the present study, mechanical properties of $\mathrm{Cu}-\mathrm{Zr}$ nanoglasses and $\mathrm{Fe}-\mathrm{Sc}$ nanocomposites were investigated by means of micro-compression tests. An improvement in plasticity was demonstrated in both $\mathrm{Cu}-\mathrm{Zr}$ nanoglasses and $\mathrm{Fe}-\mathrm{Sc}$ nanocomposites compared to conventional rapidly quenched glasses. Both the nanoglass and the composite deformed by the generation of multiple shear bands resulting in enhanced plasticity. Yield strength was also found to be high in $\mathrm{Cu}-\mathrm{Zr}$ nanoglasses compared to the conventional metallic glasses. In conclusion, compositionally tailoring such nanostructures by suitable chemistry and using such bottom-up approach synthesis techniques, better mechanical properties can be realized.

\section{Materials and methods}

In the present work, inert gas condensation (IGC) was used to synthesize $\mathrm{Cu}_{50} \mathrm{Zr}_{50}$ and $\mathrm{Fe}_{90} \mathrm{Sc}_{10}$ metallic nanoglasses. The chamber was initially evacuated to a pressure of $10^{-8} \mathrm{mbar}$ and then back filled with an inert gas. Working pressure in the range of $10^{-1}$ mbar was used to synthesize amorphous nanoparticles, which are subsequently consolidated in situ by applying a pressure of up to 1.5-3 GPa. Further compaction is performed by applying a higher pressure of up to $6 \mathrm{GPa}$. In the case of $\mathrm{Cu}_{50} \mathrm{Zr}_{50}$ alloy, magnetron sputtering was used to ablate the metal atoms, while thermal evaporation was used to synthesize $\mathrm{Fe}_{90} \mathrm{Sc}_{10}$ nanoparticles. To perform magnetron sputtering, an alloy target of $\mathrm{Cu}_{50} \mathrm{Zr}_{50}$ was used in a continuous flow of Ar and He gases and a power of $420 \mathrm{~W}$ was used with currents of about 1.9 A to extract the metal atoms. In order to synthesize using thermal evaporation, a $\mathrm{Fe}_{86} \mathrm{Sc}_{14}$ alloy was initially made by arc melting of $\mathrm{Fe}$ and Sc pieces using a Edmund Buehler arc melter in Ar atmosphere. Then the alloy is resistively heated in a tungsten boat in He atmosphere in an IGC to synthesize nanoparticles. Similar vapour pressures of Fe and Sc helps in obtaining the final powder with a uniform composition during thermal evaporation in IGC. To know further details about the synthesis procedure, the reader is requested to go through the following references [5, 10]. Ribbons of the same composition produced by standard melt-spinning technique were used as reference samples.

Preliminary characterization was performed by X-ray diffraction (XRD) using a Phillips Mo Ka X-ray source to determine the amorphous nature. To investigate the presence of nanocrystallinity in the samples, transmission electron microscopy (TEM) was performed using a TITAN 80-300 microscope (FEI Company, Hillsboro, OR, USA) operated at $300 \mathrm{kV}$, equipped with a US 1000 (Gatan Inc, Pleasanton, CA USA) slowscan CCD camera and a high-angle annular dark field (HAADF) detector (Fischione Inc, Export, PA, USA). Focused ion beam (FIB) milling in a Dual Beam Strata $400 \mathrm{~S}$ (FEI Company, Hillsboro, OR, USA) was used to prepare the lamellae for TEM observations. APT was performed using a Cameca-LEAP 4000X HR instrument in laser pulsing mode. The reconstruction of the APT data was performed with IVAS 3.6.14. To perform the micro-compression tests, both the metallic glass and nanoglasses were glued to an aluminum sample holder using superglue. Subsequently, the pillars were machined using FIB milling in a Dual Beam Strata 400 and Scios Nanolab 200 (both FEI Company, Hillsboro, OR, USA). Prior to the FIB milling, a $100 \mathrm{~nm}$ thick layer of Platinum was deposited using the electron beam to protect the surface during the milling process. The pillars were machined in four stages with decreasing ion beam currents to limit the damage caused by $\mathrm{Ga}^{+}$ions. All the $\mathrm{Fe}-\mathrm{Sc}$ and $\mathrm{Cu}-\mathrm{Zr}$ pillars had an aspect ratio of height-to-diameter of $\sim 2: 1$ and $\sim 3.5: 1$, respectively. Diameter of all the pillars is approximately $1 \mu \mathrm{m}$, as shown in Fig. 1. The taper of the pillars due to annular milling was less than $3^{\circ}$. Microcompression testing of the pillars was carried out in a nanoindenter G200 XP (Agilent/Keysight Technologies, Inc., Santa Rosa, CA, USA) with a flat punch of $10 \mu \mathrm{m}$ in diameter. Selected FeSc nanoglass pillars were deformed in situ in the SEM using an InSem nanoindenter (Nanomechanics, Inc., Oak Ridge, TN, USA). Micro-pillars were tested at different displacement rates to understand their deformation behavior. The images of the post-deformed pillars were obtained by scanning electron microscopy (SEM, Dual Beam Strata 400, FEI Company, Hillsboro, OR, USA). 


\section{Acknowledgments}

S.H.N and H.H would like to thank the DFG/SPP 1594 program for funding the project under HA1344/30-2. H.H. and Y.I. acknowledge the international cooperation possible by project HA1344/38-1. S.H.N acknowledges Dr. Zbigniew Sniadecki, Institute of Molecular Physics, Poznan for providing the $\mathrm{Cu}-\mathrm{Zr}$ metallic glasses and Dr. Di Wang, Institute of Nanotechnology, Karlsruhe Institute of Technology (KIT) for performing TEM measurements on $\mathrm{Cu}-\mathrm{Zr}$ nanoglasses. S.H.N acknowledges Dr. Reda Chellali, Institute of Nanotechnology, Karlsruhe Institute of Technology (KIT) for performing APT measurements. C.W. and Y.I. acknowledge the Research Grant from Jiangxi Provincial Department of Science and Technology under contract No.20203BDH80W008. We would also like to acknowledge the contributions of the late Dr. Oliver Franke, Viterbi School of Engineering, University of Southern California. Our friend and colleague passed away unexpectedly before the work reached its conclusion.

\section{Funding}

Open Access funding enabled and organized by Projekt DEAL.

\section{Data availability}

The datasets generated during and/or analyzed during the current study are available from the corresponding author on reasonable request.

\section{Declarations}

Conflict of interest The authors declares that they have no conflict of interest.

\section{Open Access}

This article is licensed under a Creative Commons Attribution 4.0 International License, which permits use, sharing, adaptation, distribution and reproduction in any medium or format, as long as you give appropriate credit to the original author(s) and the source, provide a link to the Creative Commons licence, and indicate if changes were made. The images or other third party material in this article are included in the article's Creative Commons licence, unless indicated otherwise in a credit line to the material. If material is not included in the article's Creative Commons licence and your intended use is not permitted by statutory regulation or exceeds the permitted use, you will need to obtain permission directly from the copyright holder. To view a copy of this licence, visit http://creativecommons.org/ licenses/by/4.0/.

\section{References}

1. J. Jing, A. Kramer, R. Birringer, H. Gleiter, U. Gonser, Modified atomic structure in a Pd-Fe-Si nanoglass. J. Non. Cryst. Solids. 113, 167-170 (1989)

2. Y. Ivanisenko, C. Kubel, S.H. Nandam, C. Wang, X. Mu, O. Adjaoud, K. Albe, H. Hahn, Structure and properties of nanoglasses. Adv. Eng. Mater. (2018). https://doi.org/10.1002/adem. 201800404

3. H. Gleiter, Nanocrystalline materials. Prog. Mater. Sci. 33, 223-315 (1989). https://doi.org/10.1016/0079-6425(89)90001-7

4. H. Gleiter, Our thoughts are ours, their ends none of our own: are there ways to synthesize materials beyond the limitations of today? Acta Mater. 56, 5875-5893 (2008). https://doi.org/10. 1016/j.actamat.2008.08.028

5. R. Witte, T. Feng, J.X. Fang, A. Fischer, M. Ghafari, R. Kruk, R.A. Brand, D. Wang, H. Hahn, H. Gleiter, Evidence for enhanced ferromagnetism in an iron-based nanoglass. Appl. Phys. Lett. 103, 073106 (2013). https://doi.org/10.1063/1.4818493

6. M.R. Chellali, S.H. Nandam, S. Li, M.H. Fawey, E. MorenoPineda, L. Velasco, T. Boll, L. Pastewka, R. Kruk, P. Gumbsch, H. Hahn, Amorphous nickel nanophases inducing ferromagnetism in equiatomic Ni-Ti alloy. Acta Mater. (2018). https://doi.org/10. 1016/j.actamat.2018.09.019

7. N. Chen, X. Shi, R. Witte, K.S. Nakayama, K. Ohmura, H. Wu, A. Takeuchi, H. Hahn, M. Esashi, H. Gleiter, A. Inoue, D.V. Louzguine, A novel Ti-based nanoglass composite with submicron-nanometer-sized hierarchical structures to modulate osteoblast behaviors. J. Mater. Chem. B. 1, 2568 (2013). https:// doi.org/10.1039/c3tb20153h

8. S. Bag, A. Baksi, S.H. Nandam, D. Wang, X. Ye, J. Ghosh, T. Pradeep, H. Hahn, Nonenzymatic glucose sensing using Ni 60 $\mathrm{Nb} 40$ nanoglass. ACS Nano 14, 5543-5552 (2020). https://doi. org/10.1021/acsnano.9b09778.s001

9. J.Q. Wang, N. Chen, P. Liu, Z. Wang, D.V. Louzguine-Luzgin, M.W. Chen, J.H. Perepezko, The ultrastable kinetic behavior of an Au-based nanoglass. Acta Mater. 79, 30-36 (2014). https:// doi.org/10.1016/j.actamat.2014.07.015

10. S.H. Nandam, Y. Ivanisenko, R. Schwaiger, Z. Śniadecki, X. Mu, D. Wang, R. Chellali, T. Boll, A. Kilmametov, T. Bergfeldt, H. Gleiter, $\mathrm{H}$. Hahn, $\mathrm{Cu}-\mathrm{Zr}$ nanoglasses: atomic structure, thermal stability and indentation properties. Acta Mater. 136, 181-189 (2017). https://doi.org/10.1016/j.actamat.2017.07.001

11. S.H. Nandam, O. Adjaoud, R. Schwaiger, Y. Ivanisenko, M.R. Chellali, D. Wang, K. Albe, H. Hahn, Influence of topological structure and chemical segregation on the thermal and mechanical properties of Pd-Si nanoglasses. Acta Mater. 193, 252-260 (2020). https://doi.org/10.1016/j.actamat.2020.03.021 
12. T. Feng, D. Wang, C. Wang, N. Chen, H. Hahn, H. Gleiter, Generating metallic amorphous core-shell nanoparticles by a solid-state amorphization process. Part. Part. Syst. Charact. 33, 82-88 (2016). https://doi.org/10.1002/ppsc.201500184

13. X.L. Wang, F. Jiang, H. Hahn, J. Li, H. Gleiter, J. Sun, J.X. Fang, Plasticity of a scandium-based nanoglass. Scr. Mater. 98, 40-43 (2015). https://doi.org/10.1016/j.scriptamat.2014.11.010

14. F.C. Li, T.Y. Wang, Q.F. He, B.A. Sun, C.Y. Guo, T. Feng, Y. Yang, Micromechanical mechanism of yielding in dual nano-phase metallic glass. Scr. Mater. 154, 186-191 (2018). https://doi.org/ 10.1016/j.scriptamat.2018.05.050

15. S. Katnagallu, G. Wu, S.P. Singh, S.H. Nandam, W. Xia, L.T. Stephenson, H. Gleiter, R. Schwaiger, H. Hahn, M. Herbig, D. Raabe, B. Gault, S. Balachandran, Nanoglass-nanocrystal composite-a novel material class for enhanced strength-plasticity synergy. Small 2004400, 1-5 (2020). https://doi.org/10.1002/ smll.202004400

16. O. Franke, D. Leisen, H. Gleiter, H. Hahn, Thermal and plastic behavior of nanoglasses. J. Mater. Res. 29, 1210-1216 (2014). https://doi.org/10.1557/jmr.2014.101

17. O. Adjaoud, K. Albe, Interfaces and interphases in nanoglasses: Surface segregation effects and their implications on structural properties. Acta Mater. (2016). https://doi.org/10.1016/j.actamat. 2016.05.002

18. S.H. Nandam, Structure and Mechanical Properties of Metallic Nanoglasses, Ph.D. Thesis, Technische Universität, Darmstadt (2019). https://tuprints.ulb.tu-darmstadt.de/id/eprint/8702.

19. I.N. Sneddon, The relation between load and penetration in the axisymmetric boussinesq problem for a punch of arbitrary profile. Int. J. Eng. Sci. 3, 47-57 (1965). https://doi.org/10.1016/ 0020-7225(65)90019-4

20. P. Sudharshan Phani, W.C. Oliver, Critical examination of experimental data on strain bursts (pop-in) during spherical indentation. J. Mater. Res. 35, 1028-1036 (2020). https://doi.org/ $10.1557 / \mathrm{jmr} .2019 .416$

21. A. Bharathula, S.W. Lee, W.J. Wright, K.M. Flores, Compression testing of metallic glass at small length scales: effects on deformation mode and stability. Acta Mater. 58, 5789-5796 (2010). https://doi.org/10.1016/j.actamat.2010.06.054

22. D. Tönnies, R. Maaß, C.A. Volkert, Room temperature homogeneous ductility of micrometer-sized metallic glass. Adv. Mater. 26, 5715-5721 (2014). https://doi.org/10.1002/adma.201401123

23. C. Wang, X. Mu, M.R. Chellali, A. Kilmametov, Y. Ivanisenko, H. Gleiter, H. Hahn, Tuning the Curie temperature of Fe90Sc10 nanoglasses by varying the volume fraction and the composition of the interfaces. Scr. Mater. 159, 109-112 (2019). https://doi.org/ 10.1016/j.scriptamat.2018.09.025

24. Z. Han, W.F. Wu, Y. Li, Y.J. Wei, H.J. Gao, An instability index of shear band for plasticity in metallic glasses. Acta Mater. 57, 1367-1372 (2009). https://doi.org/10.1016/j.actamat.2008.11.018
25. Y.F. Gao, B. Yang, T.G. Nieh, Thermomechanical instability analysis of inhomogeneous deformation in amorphous alloys. Acta Mater. 55, 2319-2327 (2007). https://doi.org/10.1016/j. actamat.2006.11.027

26. H. Guo, P.F. Yan, Y.B. Wang, J. Tan, Z.F. Zhang, M.L. Sui, E. Ma, Tensile ductility and necking of metallic glass. Nat. Mater. 6 735-739 (2007). https://doi.org/10.1038/nmat1984

27. A. Dubach, F.H.D. Torre, J.F. Löffler, Deformation kinetics in Zrbased bulk metallic glasses and its dependence on temperature and strain-rate sensitivity. Philos. Mag. Lett. 87, 695-704 (2007). https://doi.org/10.1080/09500830701494037

28. D. Şopu, A. Stukowski, M. Stoica, S. Scudino, Atomic-level processes of shear band nucleation in metallic glasses. Phys. Rev. Lett. 119, 1-5 (2017). https://doi.org/10.1103/PhysRevLett.119. 195503

29. Y.Q. Cheng, A.J. Cao, E. Ma, Correlation between the elastic modulus and the intrinsic plastic behavior of metallic glasses: the roles of atomic configuration and alloy composition. Acta Mater. 57, 3253-3267 (2009). https://doi.org/10.1016/j.actamat.2009.03. 027

30. K.W. Park, J. Il Jang, M. Wakeda, Y. Shibutani, J.C. Lee, Atomic packing density and its influence on the properties of $\mathrm{Cu}-\mathrm{Zr}$ amorphous alloys. Scr. Mater. 57, 805-808 (2007). https://doi. org/10.1016/j.scriptamat.2007.07.019

31. R.L. Narayan, K. Boopathy, I. Sen, D.C. Hofmann, U. Ramamurty, On the hardness and elastic modulus of bulk metallic glass matrix composites. Scr. Mater. 63, 768-771 (2010). https:// doi.org/10.1016/j.scriptamat.2010.06.010

32. S.S. Hirmukhe, K.E. Prasad, I. Singh, Investigation of pressure sensitive plastic flow in nanoglasses from finite element simulations. Scr. Mater. 180, 45-50 (2020). https://doi.org/10.1016/j. scriptamat.2020.01.022

33. Z.T. Wang, J. Pan, Y. Li, C.A. Schuh, Densification and strain hardening of a metallic glass under tension at room temperature. Phys. Rev. Lett. (2013). https://doi.org/10.1103/PhysRevLett.111. 135504

34. E. Ma, Tuning order in disorder. Nat. Mater. 14, 547-552 (2015). https://doi.org/10.1038/nmat4300

35. K. Albe, Y. Ritter, D. Şopu, Enhancing the plasticity of metallic glasses: shear band formation, nanocomposites and nanoglasses investigated by molecular dynamics simulations. Mech. Mater. 67, 94-103 (2013). https://doi.org/10.1016/j.mechmat.2013.06. 004

36. F. Shimizu, S. Ogata, J. Li, Yield point of metallic glass. Acta Mater. 54, 4293-4298 (2006). https://doi.org/10.1016/j.actamat. 2006.05.024

37. D. Klaumünzer, R. Maaß, J.F. Löffler, Stick-slip dynamics and recent insights into shear banding in metallic glasses. J. Mater. Res. 26, 1453-1463 (2011). https://doi.org/10.1557/jmr.2011.178

38. P. Thurnheer, R. Maaß, K.J. Laws, S. Pogatscher, J.F. Löffler, Dynamic properties of major shear bands in $\mathrm{Zr}-\mathrm{Cu}-\mathrm{Al}$ bulk 
metallic glasses. Acta Mater. 96, 428-436 (2015). https://doi.org/ 10.1016/j.actamat.2015.05.028

39. Q. An, K. Samwer, M.D. Demetriou, M.C. Floyd, D.O. Duggins, W.L. Johnson, W.A. Goddard, How the toughness in metallic glasses depends on topological and chemical heterogeneity. Proc. Natl. Acad. Sci. 113, 7053-7058 (2016). https://doi.org/10.1073/ pnas. 1607506113

40. T. Brink, M. Peterlechner, H. Rösner, K. Albe, G. Wilde, Influence of crystalline nanoprecipitates on shear-band propagation in $\mathrm{Cu}-\mathrm{Zr}$-based metallic glasses. Phys. Rev. Appl. 5, 1-16 (2016). https://doi.org/10.1103/PhysRevApplied.5.054005

41. A. Inoue, W. Zhang, T. Tsurui, A.R. Yavari, A.L. Greer, Unusual room-temperature compressive plasticity in nanocrystaltoughened bulk copper-zirconium glass. Philos. Mag. Lett. 85 221-229 (2005). https://doi.org/10.1080/09500830500197724
42. J. Kim, H.S. Oh, W. Kim, P.P. Choi, D. Raabe, E.S. Park, Modulation of plastic flow in metallic glasses via nanoscale networks of chemical heterogeneities. Acta Mater. 140, 116-129 (2017). https://doi.org/10.1016/j.actamat.2017.08.002

43. D. Jang, J.R. Greer, Size-induced weakening and grain boundaryassisted deformation in $60 \mathrm{~nm}$ grained Ni nanopillars. Scr. Mater. 64, 77-80 (2011). https://doi.org/10.1016/j.scriptamat.2010.09. 010

44. Y. Ivanisenko, T. Werz, A. Minkow, J. Lohmiller, P.A. Gruber, A. Kobler, L. Kurmanaeva, H.J. Fecht, Observation of shear band formation in nanocrystalline Pd-Au alloy during in situ SEM compression testing. J. Mater. Sci. 48, 6841-6847 (2013). https:// doi.org/10.1007/s10853-013-7490-7 